\title{
Combined analysis of PTEN, HER2, and hormone receptors status: remodeling breast cancer risk profiling
}

Elham Sajjadi ${ }^{1,2+}$, Konstantinos Venetis ${ }^{1,2+}$, Roberto Piciotti ${ }^{1,2}$, Donatella Gambini ${ }^{3}$, Concetta Blundo ${ }^{4}$, Letterio Runza ${ }^{5}$, Stefano Ferrero ${ }^{5,6}$, Elena Guerini-Rocco ${ }^{1,2}$ and Nicola Fusco ${ }^{1,2^{*}}$

\begin{abstract}
Background: Phosphatase and tensin homolog (PTEN) loss is associated with tumorigenesis, tumor progression, and therapy resistance in breast cancer. However, the clinical value of PTEN as a biomarker in these patients is controversial. We sought to determine whether the benefit of traditional biomarkers testing is improved by the analysis of PTEN status for the identification of high-risk breast cancer.
\end{abstract}

Methods: A cohort of 608 patients with breast cancer was included in this study. Based on the expression on the neoplastic cells compared to the normal internal controls by immunohistochemistry $(\mathrm{IHC})$, cases were classified as PTEN-low (PTEN-L) or PTEN-retained (PTEN-WT). The former constituted the study group, while the latter the control group. Analysis of gene expression was performed on publicly available genomic data and included 4265 patients from the METABRIC and MSK cohorts retrieved from cBioPortal. The Shapiro-Wilk test was used to analyze the normal distributions of continuous variables. Relationships between PTEN status and the clinicopathologic and molecular features of the patient population were assessed using Fisher's exact test or Chi-squared/Wilcoxon ranksum test. Survival curves were built according to the Kaplan-Meier method.

Results: Alteration in PTEN status was significantly different at protein and gene levels, where the reduced protein expression was observed in 280/608 cases (46.1\%) from our group, while genetic aberrations in only 315/4265 (7.4\%) cases of the METABRIC and MSK cohorts. PTEN-L tumors were significantly enriched for hormone receptors (HR) and HER2 negativity $(n=48,17.1 \%)$ compared to PTEN-WT tumors $(n=22,6.7 \% ; p=0.0008)$. Lack of HR with or without HER2 overexpression/amplification was significantly associated with worse overall survival (OS) in PTEN-L but not in PTEN-WT breast cancers ( $p<.0001)$. Moreover, PTEN-L protein expression but not gene alterations was related to the outcome, in terms of both OS and disease-free survival $(p=0.002)$.

Conclusions: The combined analysis of PTEN, HER2, and HR status offers relevant information for a more precise risk assessment of patients with breast cancer.

Keywords: PTEN, Hormone receptors, Estrogen receptor, Progesterone receptor, HER2, Breast cancer, Prognosis, Biomarkers

\footnotetext{
* Correspondence: nicola.fusco@unimi.it

${ }^{\dagger}$ Elham Sajjadi and Konstantinos Venetis contributed equally to this work.

${ }^{1}$ Department of Oncology and Hemato-Oncology, University of Milan, Via Festa del Perdono 7, 20122 Milan, Italy

${ }^{2}$ Division of Pathology, IEO, European Institute of Oncology IRCCS, Via Giuseppe Ripamonti 435, 20141 Milan, Italy

Full list of author information is available at the end of the article
}

C C The Author(s). 2021 Open Access This article is licensed under a Creative Commons Attribution 4.0 International License, which permits use, sharing, adaptation, distribution and reproduction in any medium or format, as long as you give appropriate credit to the original author(s) and the source, provide a link to the Creative Commons licence, and indicate if changes were made. The images or other third party material in this article are included in the article's Creative Commons licence, unless indicated otherwise in a credit line to the material. If material is not included in the article's Creative Commons licence and your intended use is not permitted by statutory regulation or exceeds the permitted use, you will need to obtain permission directly from the copyright holder. To view a copy of this licence, visit http://creativecommons.org/licenses/by/4.0/ The Creative Commons Public Domain Dedication waiver (http://creativecommons.org/publicdomain/zero/1.0/) applies to the data made available in this article, unless otherwise stated in a credit line to the data. 


\section{Background}

Phosphatase and tensin homolog (PTEN) is a tumor suppressor and negative regulator of the phosphatidylinositol3-kinase $(\mathrm{PI} 3 \mathrm{~K}) /$ protein kinase $\mathrm{B}$ (Akt) pathway $[1,2]$. Loss of PTEN activity has been reported across a variety of primary and metastatic malignancies, including breast cancer, and is related to tumorigenesis, tumor progression, and therapy resistance [3-5].

In breast cancer, the clinical actionability of PTEN status has been studied in both prognostic and predictive settings [6, 7]. Alterations of PTEN and the serine/threonine kinase Akt isoforms have been observed in patients with HER2 + breast cancers with a low response to trastuzumab therapy [8]. Additionally, analysis of PTEN expression has been proposed as a complementary biomarker for mismatch repair status assessment in breast cancer, potentially contributing to the selection of patients, including those with a hormone receptor (HR) + tumor, eligible to immune-checkpoint blockade $[4,9]$. Notably, the phase III Breast Cancer International Research Group (BCIRG)-006 trial demonstrated that PTEN loss is linked to a worse prognosis but not to trastuzumab resistance in patients with HER2+ breast cancer [10]. A meta-analysis of 27 studies including 10,231 breast cancers, further provided evidence that PTEN loss might be a predictor of aggressive behavior [11]. On the other hand, recent clinical and translational studies failed to identify a significant association between PTEN status and patients' outcomes [12, 13]. So, the consistency of PTEN testing in clinical practice for patients with breast cancer remains unclear [14].

We hypothesized that, if alterations in PTEN have potent pro-oncogenic activity in breast cancer, detailed information on the status of this tumor suppressor could be used to improve clinical trial design and patients' clinical management. In this study, we provide insights on the patterns of PTEN alterations along with HER2 and HR status in different groups of breast cancer and identify novel significant subsets of patients with highrisk neoplasms.

\section{Materials and methods}

\section{Patients and tissue specimens}

This study was approved by the local Ethical Committee under protocol number \#620_2018bis. All patients included in this study were diagnosed and managed at the Fondazione IRCCS Ca' Granda - Ospedale Maggiore Policlinico between 2004 and 2018, and they are part of an anonymized database encompassing detailed clinicopathologic and follow-up data [15]. For this study, patients were included based on the availability of formalin-fixed, paraffin-embedded (FFPE) and frozen tissue. All cases were reviewed, re-classified, and re-graded according to the latest World Health Organization
(WHO) recommendations [16] and the Nottingham histologic grading system [17], respectively. Pathologic re-staging was performed following the 8th edition of the American Joint Committee on Cancer (AJCC) Cancer Staging Manual [18].

\section{Tissue microarrays construction}

Representative FFPE blocks were selected for tissue microarray (TMA) construction, as previously described [19]. For each case, the core and periphery (i.e., invasive front) of the tumor, in situ (i.e., intraductal) component (if present), and matched normal epithelial breast tissue (i.e., glandular tissue with at least one non-neoplastic terminal ductal-lobular unit adjacent to the neoplasm) were sampled. A total of 5 tumor samples and one matched normal tissue per patient, with a diameter of $1 \mathrm{~mm}$, were incorporated in the corresponding TMA block.

\section{Immunohistochemical analysis}

Four- $\mu$ m-thick sections were cut from the TMA blocks and subjected to immunohistochemistry (IHC) using anti-human pre-diluted antibodies for PTEN, estrogen receptor (ER), progesterone receptor (PgR), Ki67, and HER2 on a Dako Omnis automated staining systems (Agilent, Santa Clara, CA, USA) [20]. For each antibody, positive and negative controls were included in each slide run. HR (i.e. ER and PgR) and HER2 status were tested and reported according to the breast biomarker reporting guidelines v1.4.1.0 published by the College of American Pathologists (CAP) in June 2021 (available at https://www.cap.org/protocols-and-guidelines). The proliferation index was assessed by $\mathrm{Ki} 67 \mathrm{IHC}$ as the global (average) score across the section. According to the updated recommendations from the International Ki67 in Breast Cancer Working Group, a cut-off value of $\geq 30 \%$ was used to define the high proliferation group [21]. PTEN expression was scored using a three-tier system that considers the ratio between normal and tumor tissue, as proposed by Sakr et al. [22, 23]. Specifically, score 0 indicated the absence of staining in tumor cells but not in the surrounding normal epithelial and stromal cells, score 1 was considered when the tumor cell staining was weaker than the surrounding normal epithelial and stromal cells. In the case of staining equal to that of the normal epithelial and stromal cells, the case was scored as 2. Subsequently, PTEN status was assessed dichotomously as "low" (PTEN-L) and "wild type" (PTENWT) for cases with scores $0-1$ and score 2, respectively [4]. Details of antibodies, clones, dilutions, antigen retrieval methods, and scoring systems adopted in this study are provided in Supplementary Table S1. 


\section{cBioPortal and statistical analyses}

Clinical and genomic data were extracted from the METABRIC and the MSK datasets made available by The Cancer Genome Atlas Network (TCGA) at cBioPortal [24]. Relationships between PTEN status and the clinicopathologic and molecular features of the patients were assessed using Fisher's exact test or Chi-squared test [25]. Odds ratio (OR) and corresponding 95\% confidence interval (CI) were calculated for each variable [26-28]. To identify factors associated with PTEN expression, multinomial logistic regression models were defined considering a stepwise selection procedure [29]. Survival curves were built according to the Kaplan-Meier method and compared using the log-rank test [30]. All statistical tests were two-tailed; $p$-values $<0.05$ were considered statistically significant; reported $p$-values were not corrected for multiple testing.

\section{Results}

A total of 608 patients with invasive breast cancer (age, 26-92 years; mean, 61.0 (12.9) years) diagnosed between 2004 and 2018 were included in this study (follow-up time, 1-172 months; mean, 57.8 (50.1) months). Their demographic and clinicopathologic characteristics are listed in Table 1. Follow-up data were available for 603 (99\%) patients.

Decreased PTEN protein expression is more frequent than gene alterations in breast cancer

Taken together, $46.1 \%(n=280 / 608)$ cases showed a decreased or null expression of the PTEN protein by IHC, as depicted in Fig. 1, and were therefore classified as PTEN-L. Conversely, analysis of the genomic data from the METABRIC and MSK portal cohorts including 4265 patients, revealed mutations, deep deletions, fusions, and/or amplifications in PTEN in only 315 (7.4\%) patients (Fig. 2). These data suggest that, in breast cancer, alterations targeting PTEN are common events that more likely occur after transcription.

\section{The spectrum of PTEN alterations varies across different breast cancer subtypes}

Among the patients included in this study, the mean age at diagnosis was similar in the PTEN-L (61.5 years) and PTEN-WT (60.6 years subgroups). In the former group, ductal $(n=224,80 \%)$ and HR+/HER2- $(n=213,76.1 \%)$ were the most frequent histological and molecular subtypes, respectively. Additionally, high grade $(n=130$, $46.4 \%)$, and low stage $(n=118,42.1 \%)$ tumors were more prevalent in the PTEN-L subgroup, as detailed in Fig. 1 and Table 2. Considering the PTEN intra-tumor expression pattern, the spatial distribution and IHC staining intensity were homogeneous, as demonstrated

Table 1 Clinicopathologic features of the patients included in this study according to their biomarker status

\begin{tabular}{|c|c|c|c|c|}
\hline & HR+/HER2- & HER2+ & HR-/HER2- & Total \\
\hline All patients, $n(\%)$ & $488(80)$ & $50(8)$ & $70(12)$ & $608(100)$ \\
\hline \multicolumn{5}{|l|}{ Age, $n(\%)$} \\
\hline$\geq 55$ years & $350(83)$ & $32(8)$ & $38(9)$ & $420(69)$ \\
\hline$<55$ years & $138(73)$ & $18(10)$ & $32(17)$ & $188(31)$ \\
\hline \multicolumn{5}{|l|}{ Menopause, $n$ (\%) } \\
\hline Yes & $383(82)$ & $41(9)$ & $44(9)$ & $468(77)$ \\
\hline No & $103(76)$ & $8(6)$ & $25(18)$ & $136(22)$ \\
\hline $\mathrm{n} / \mathrm{a}$ & $2(50)$ & $1(25)$ & $1(25)$ & $4(1)$ \\
\hline
\end{tabular}

Histology, $n(\%)$

$\begin{array}{lllll}\text { Ductal } & 365(79) & 45(10) & 52(11) & 462(76) \\ \text { Lobular } & 71(92) & 3(4) & 3(4) & 77(13) \\ \text { Other } & 52(75) & 2(3) & 15(22) & 69(11)\end{array}$

Grade, $n$ (\%)

2

3

68 (94)

1 (1) $3(4)$

72 (12)

240 (93)

11 (4) $7(3)$

258 (42)

R, $n$ (\%)

Positive

488 (92)

40 (8) $0(0)$

528 (87)

Negative

$0(0)$

10 (13) $\quad 70$ (88)

80 (13)

PgR, n (\%)

Positive

418 (93)

$30(7) \quad 0(0)$

$448(73)$

Negative

$70(44)$

$20(12) \quad 70(44)$

$160(27)$

HER2, $n$ (\%)

Positive

$0(0)$

$50(100)$

$0(0)$

50 (8)

Negative

488 (87)

$0(0)$

70 (13)

$558(92)$

Ki67, n (\%)

High

Low

Stage, $n(\%)$

।

II

III-IV

$\mathrm{n} / \mathrm{a}$

Molecular subtype, $n(\%)$

\begin{tabular}{lllll} 
Luminal $^{\mathrm{a}}$ & $204(100)$ & $0(0)$ & $0(0)$ & $204(38)$ \\
Luminal $^{\mathrm{b}}$ & $284(88)$ & $40(12)$ & $0(0)$ & $324(49)$ \\
HER2-type1 $^{\mathrm{c}}$ & $0(0)$ & $10(100)$ & $0(0)$ & $10(2)$ \\
TNBC $^{\mathrm{d}}$ & $0(0)$ & $0(0)$ & $70(100)$ & $70(11)$ \\
\hline
\end{tabular}

HR Hormone receptors, ER Estrogen receptor, $P g R$ Progesterone receptor, $T N B C$ Triple-negative breast cancer, $n / a$ not available; ${ }^{a} E R+/ P R+/ K i 67$ low; ${ }^{b} E R+/ K i 67$ high or ER+/PR-; ${ }^{\mathrm{C} E R-/ P R-/ H E R 2+; ~}{ }^{\mathrm{d} E R-/ P R-/ H E R 2-~}$

by the analysis of full sections in PTEN-L cases. On the other hand, a high degree of inter-tumor heterogeneity was observed, with a significant association between 


\section{PTEN-L $(n=280)$}

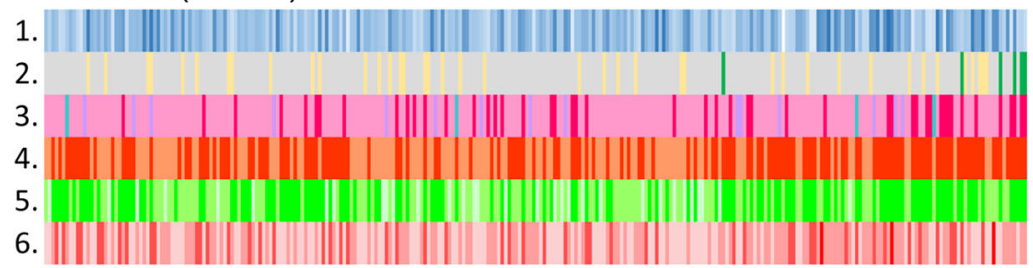

PTEN-WT $(n=328)$
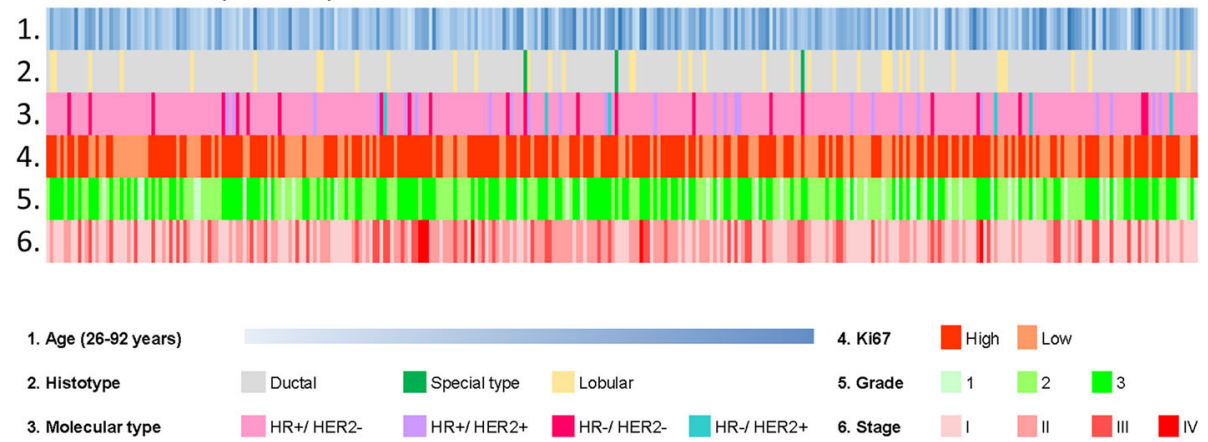

Fig. 1 Heatmap illustrating selected clinicopathologic features of the patients included in this study according to the PTEN status. Each column represents a patient, each row a parameter, color-coded according to the legend below. PTEN-L, PTEN low (i.e. decreased expression); PTEN-WT, PTEN wild-type (i.e. retained expression) HR, hormone receptors

PTEN and HR/HER2 status ( $p=0.0008$ according to the chi-square independence test), as shown in Table 2). More in detail, in the PTEN-L population, the prevalence of HR-/HER2- tumors $(n=48,17.1 \%)$ was higher compared to the PTEN-WT group $(n=22,6.7 \%)$. These findings suggest that PTEN loss or reduced expression is a more common event in tumors lacking the HR and/or HER2 expression/amplification.
PTEN status assessment improves HR and HER2 prognostic value

Overall, a higher rate of patients died of disease in the PTEN-L population ( $n=26,9.4 \%$, PTEN-L versus $n=8$, $2.5 \%$, PTEN-WT; $p=0.0001$ ), particularly in HR-/HER2$(p=0.0006)$ and locally advanced or metastatic breast cancers $(p=0.0006)$, as shown in Table 3 . According to our multivariable model, PTEN status was an independent

Fig. 2 Oncoprint visualization of genetic alterations (i.e., inframe, missense, splice and truncating mutations, fusions, amplifications, and deep deletions) of the PTEN gene in breast cancer. In this analysis involving two different datasets available at cbioportal.org (patients $n=4265$; samples $n=4427$ ), truncating mutations and deep deletions were the most frequent alterations. Taken together, in 315 (7.4\%) of the patients the queried gene was found to be altered. Each column represents a patient/sample and was sorted for the magnitude of alteration types in the queried genes. The types of alterations and the study of origin are color-coded as shown in the legend; the blue and red rectangles refer to the METABRIC and MSK studies, respectively
} 
Table 2 Correlation between low and wildtype status of PTEN across selected clinicopathologic features

\begin{tabular}{|c|c|c|c|}
\hline & PTEN-L & PTEN-WT & $p$-value \\
\hline All patients, n (\%) & $280(46.1)$ & $328(53.9)$ & \\
\hline Age, mean (SD) & $61.5(12.0)$ & $60.6(13.6)$ & 0.3425 \\
\hline \multicolumn{4}{|l|}{ Histology, n (\%) } \\
\hline Ductal & $224(80.0)$ & $267(81.4)$ & \multirow[t]{3}{*}{0.6243} \\
\hline Lobular & $40(14.3)$ & 39 (11.9) & \\
\hline Other & $16(5.7)$ & $22(6.7)$ & \\
\hline \multicolumn{4}{|c|}{ HR and HER2 status, n (\%) } \\
\hline $\mathrm{HR}+/$ HER2- & $213(76.1)$ & $275(83.8)$ & \multirow[t]{4}{*}{0.0008} \\
\hline HR-/ HER2+ & $4(1.4)$ & $6(1,8)$ & \\
\hline $\mathrm{HR}+/$ HER2+ & $15(5.4)$ & $25(7.6)$ & \\
\hline HR-/ HER2- & $48(17.1)$ & $22(6.7)$ & \\
\hline \multicolumn{4}{|l|}{ Grade, n (\%) } \\
\hline 1 & $34(12.1)$ & 39 (11.9) & \multirow[t]{3}{*}{0.6428} \\
\hline 2 & $116(41.4)$ & $148(45.1)$ & \\
\hline 3 & $130(46.4)$ & $141(43.0)$ & \\
\hline \multicolumn{4}{|l|}{$\mathrm{T}, \mathrm{n}(\%)$} \\
\hline 1 & $180(64.3)$ & $198(60.4)$ & \multirow[t]{4}{*}{0.7479} \\
\hline 2 & $83(29.6)$ & $105(32.0)$ & \\
\hline 3 & $6(2.1)$ & $8(2.4)$ & \\
\hline 4 & $11(3.9)$ & $17(5.2)$ & \\
\hline \multicolumn{4}{|l|}{ N, n (\%) } \\
\hline Positive & $164(58.6)$ & $207(63.1)$ & \multirow[t]{2}{*}{0.2528} \\
\hline Negative & $116(41.4)$ & $121(36.9)$ & \\
\hline \multicolumn{4}{|l|}{ Stage, n (\%) } \\
\hline 0,1 & $118(42.1)$ & $154(47.0)$ & \multirow[t]{3}{*}{0.4682} \\
\hline 2 & $107(38.2)$ & $112(34.2)$ & \\
\hline 3,4 & 55 (19.6) & $62(18.9)$ & \\
\hline
\end{tabular}

PTEN-L PTEN low (i.e. decreased expression), PTEN-WT PTEN wild-type (i.e. retained expression) $H R$ Hormone receptors. SD Standard deviation

predictor of both death for disease and disease recurrence. In particular, loss of expression was significantly associated with patients' death $(p=0.001)$ and the presence of unfavorable prognostic factors, such as triple-negative $(p=0.002)$ and HER2+ $(p<0.0001)$ phenotypes, as depicted in Table 4. Not surprisingly, patients with HR-/HER2- breast cancer harbored a high risk of death $(n=10,14.3 \% ; p=0.0006)$ (Table 3$)$. Of note, in PTEN-L tumors, HR-/HER2+ and HR+/HER2+ clusters showed an increased death prevalence $(n=$ $1,25.0 \%$ and $n=4,26.7 \%$, respectively) compared to the HR-/HER2- and HR+/HER2- $(n=4,8.3 \%$ and $n=13,6.1 \%$, respectively; $p=0.02$ ) (Fig. 3, Supplementary Table S2 and Table S3). These analyses provide evidence that PTEN loss or reduced expression is a bona fide prognostic parameter in breast cancer.
Table 3 Bivariate analysis showing the association of selected clinicopathologic characteristics with patients' death

\begin{tabular}{llll}
\hline & \multicolumn{2}{l}{ Death } & \\
\cline { 2 - 4 } & Yes & No & $\boldsymbol{p}$-value \\
\hline HR and HER2 status, $\boldsymbol{n}(\%)$ & & 0.0006 \\
HR+/ HER2- & $18(3.7)$ & $465(96.3)$ & \\
HR-/ HER2+ & $1(10.0)$ & $9(90.0)$ & \\
HR+/ HER2+ & $5(12.0)$ & $35(88.0)$ & \\
HR-/ HER2- & $10(14.3)$ & $60(85.7)$ &
\end{tabular}

Stage, $\mathbf{n}(\%)$

0.0006

$\begin{array}{lll}0,1 & 8(3.0) & 263(97.0) \\ 2 & 12(5.6) & 204(94.4) \\ 3,4 & 15(12.9) & 101(87.1)\end{array}$

PTEN status

0.0001

$\begin{array}{lll}\text { PTEN-WT, n (\%) } & 8(2.5) & 317(97.5) \\ \text { PTEN-L, n (\%) } & 26(9.4) & 252(90.6)\end{array}$

Death status was available for 603 patients. PTEN-L PTEN low (i.e. decreased expression), PTEN-WT PTEN wild-type (i.e. retained expression), HR Hormone receptors

Shorter survival in HR-negative PTEN-L breast cancers Survival analysis stratified for PTEN status (Fig. 4, Supplementary Fig. S1, and Supplementary Fig. S2) showed that, patients with PTEN-L $(p=0.03)$ but not PTENWT $(p=0.61)$ and HR-/HER2+ breast cancer have a shorter survival probability compared to HR+/HER2(Fig. 4a). This correlation was not retained while analyzing the risk of recurrence, which was not statistically significant in both PTEN-L and PTEN-WT groups (Supplementary Fig. S2a). Given the low number of patients with $\mathrm{HR}+/ \mathrm{HER} 2+$ breast cancers in both PTEN cohorts, we further assessed HR negativity and HER2

Table 4 Multivariable analysis showing the association of selected clinicopathologic characteristics with PTEN status

\begin{tabular}{|c|c|c|c|}
\hline \multicolumn{4}{|l|}{ PTEN-WT vs. PTEN-L } \\
\hline & OR & $95 \% \mathrm{Cl}$ & $p$-value \\
\hline \multicolumn{4}{|l|}{ Death } \\
\hline Survived vs. Deceased & 0.25 & $0.11-0.55$ & 0.001 \\
\hline \multicolumn{4}{|l|}{ Grade } \\
\hline $1 / 2$ vs. 3 & 0.853 & $0.62-1.18$ & 0.329 \\
\hline \multicolumn{4}{|l|}{ HR and HER2 status } \\
\hline HR+/HER2- vs. HR+/HER2+ & 0.78 & $0.4-1.51$ & 0.451 \\
\hline HR-/HER2- vs. HR+/HER2+ & 0.275 & $0.122-0.621$ & 0.002 \\
\hline HR-/HER2+ vs. HR+/HER2+ & 0.9 & $0.22-3.72$ & 0.884 \\
\hline HR-/HER2- vs. HR+/HER2- & 0.36 & $0.21-0.61$ & $<0.0001$ \\
\hline HR-/HER2+ vs. HR+/HER2- & 1.16 & $0.32-4.17$ & 0.818 \\
\hline HR-/HER2+ vs. HR-/HER2- & 3.27 & $0.84-12.78$ & 0.09 \\
\hline
\end{tabular}

PTEN-L PTEN low (i.e. decreased expression), PTEN-WT PTEN wild-type (i.e. retained expression), $H R$ Hormone receptors, OR Odds ratio, $\mathrm{Cl}$ Confidence interval 


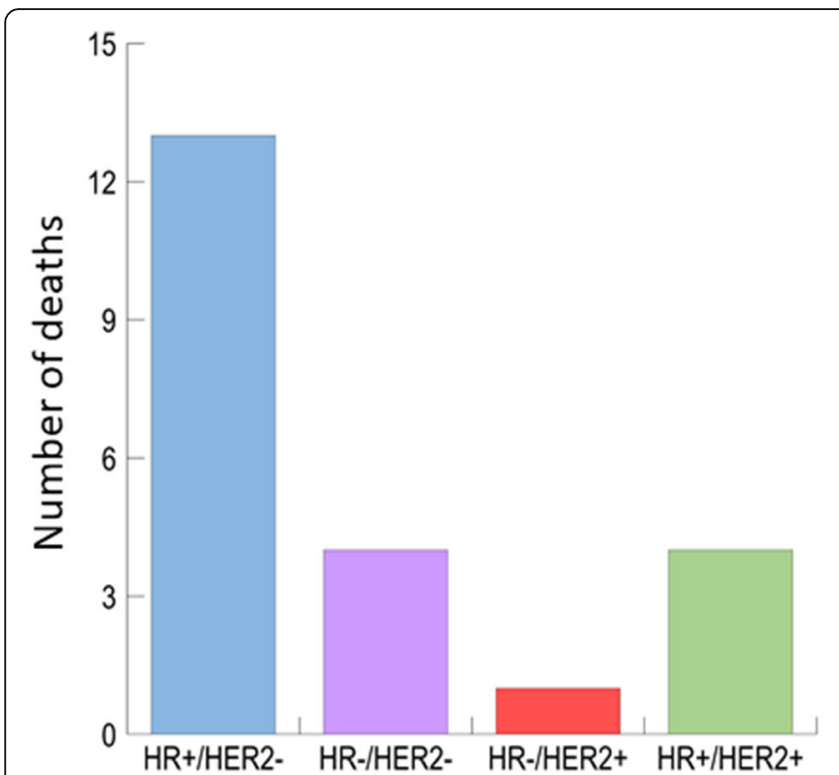

(a)

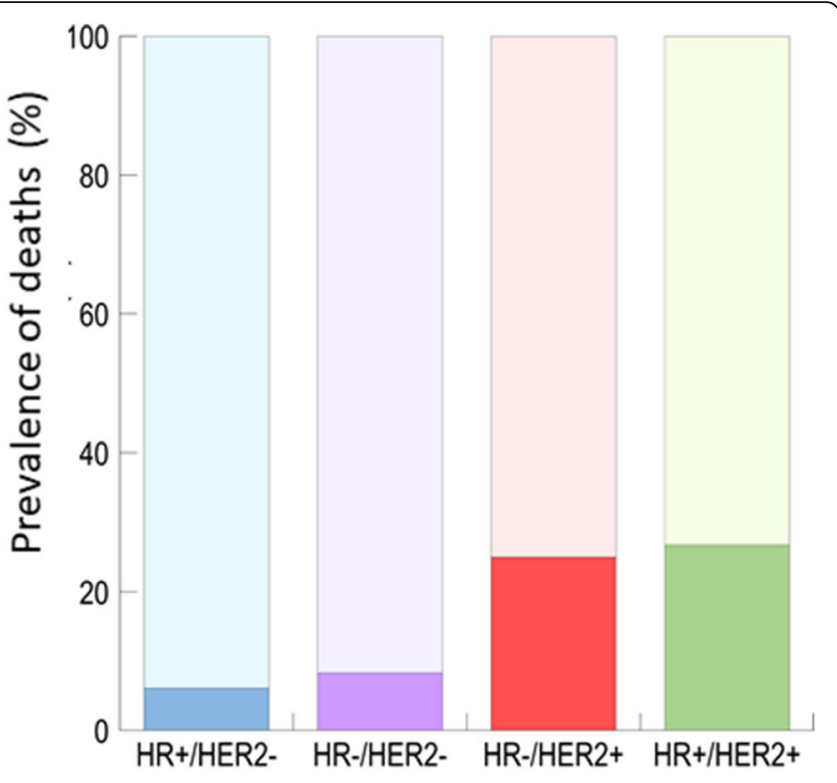

(b)

Fig. 3 Frequency (a) and proportion (b) of death across PTEN-L breast cancer subgroups. ER, estrogen receptor; PTEN, phosphatase and tensin homolog

positivity, as solo, along with PTEN status (Fig. 4b, c and Supplementary Fig. S2b, c). In both cases, the OS but not the disease-free survival was worse in PTEN-L compared to PTEN-WT neoplasms $(p<0.001$ vs $p=0.06$ and $p<0.001$ vs. $p=0.73$ ). Moreover, we observed that HER2 positivity either alone, or alongside with HR positivity, was associated with an increased risk of death in PTEN-L breast cancers $(p<0.0001$ and $p=0.002$, respectively), as shown in Fig. 4. Accordingly, none of these conditions were related to a worse prognosis when the expression of PTEN was retained $(p=0.73$ and $p=$ 0.52 , respectively). Taken together, these findings suggest that patients with HR- and/or HER2+ breast cancer have an unfavorable prognosis in terms of OS in the presence of low or null expression of PTEN but not if PTEN expression is retained.

\section{Discussion}

Here, we analyzed the PTEN status to assess its usefulness for the refining of breast cancer risk profiles in combination with traditional biomarkers testing. Our analyses show that a decreased expression of PTEN at the protein level occurs in almost half of patients, with the highest frequency in ductal and HR+ breast cancers. On the other hand, alterations of gene expression are present in the minority of patients, as previously reported [31]. The different frequency of PTEN protein and gene alterations can be due to the several regulatory layers that mediate PTEN function, including transcriptional (e.g. epigenetic mechanisms and transcription factors), post-transcriptional (e.g. miRNAs, PTEN pseudogene), and post-translational mechanisms (e.g. phosphorylation, acetylation, ubiquitination, etc.) $[6,32$, 33]. Furthermore, we confirm that in breast cancer a wide spectrum PTEN expression patterns can be observed, emphasizes the need for the implementation of well-defined IHC guidelines [34, 35].

Despite their well-defined predictive role for endocrine therapy $[25,36]$, HR have been traditionally considered weak prognostic biomarkers in breast cancer [37]. On the other hand, previous studies on endometrial cancer and pancreatic neuroendocrine tumors have unraveled a rationale for the combined assessment of HR and PTEN for patients' risk stratification [38, 39]. We found that PTEN expression levels show a statistically significant correlation with HR and HER2 status in breast cancer. Specifically, the prevalence of the HR-/HER2- subgroup, was more than double in the PTEN-L compared to PTEN-WT group. Furthermore, we confirm a higher incidence of death and disease recurrence in the PTEN-L population. Notably, the combined assessment of PTEN with HR and HER2 status showed more precise risk profiles.

When PTEN expression was low, ER negativity and HER2 positivity were related to worse OS compared to the HR+/HER2- subgroup. Moreover, in the PTEN-L but not in the PTEN-WT population, the HR+/HER2+ subgroup showed a statistically significant worse OS compared to the HR+/HER2- cluster. One of the possible explanations for the different risks observed in HR- 


\section{PTEN-L}
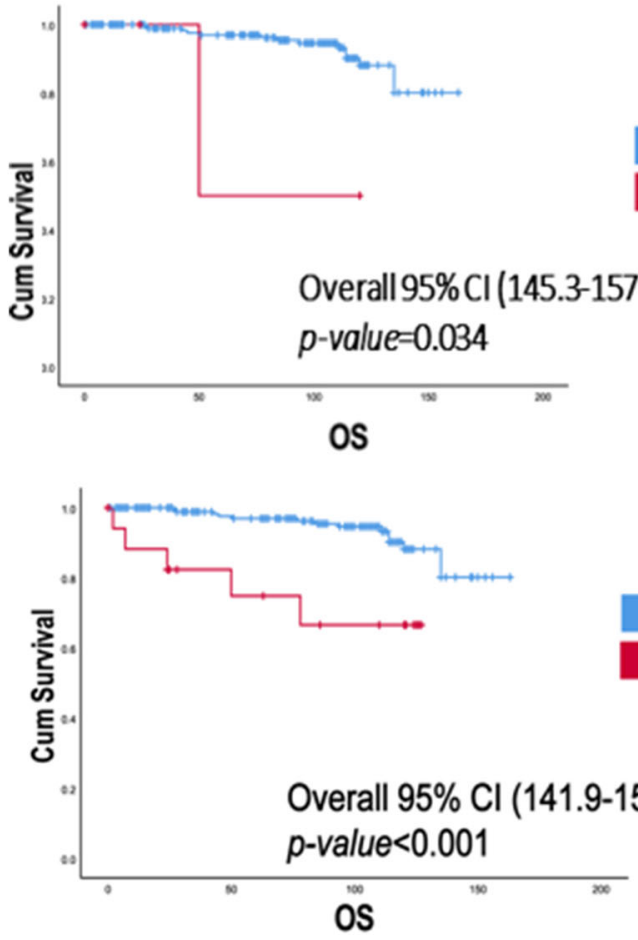

(b)

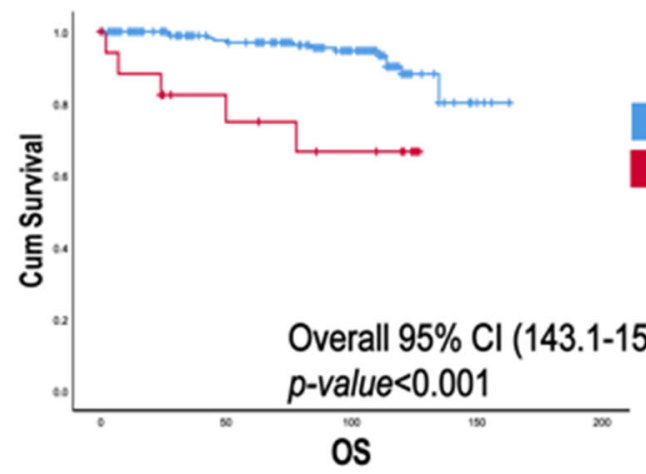

(c)

(a)

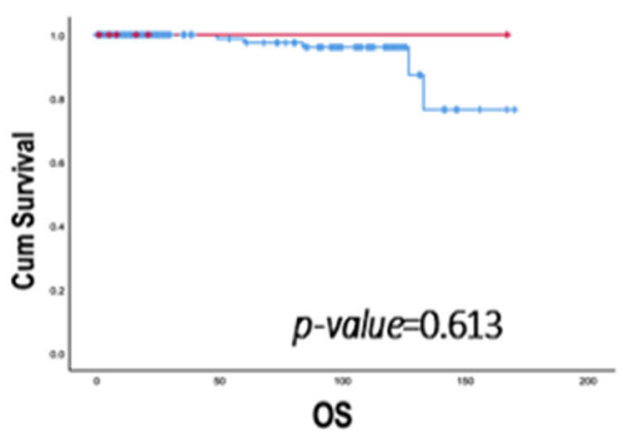

$\mathrm{HR}+/ \mathrm{HER} 2$

$\mathrm{HR}$

$\mathrm{HR}+/ \mathrm{HER} 2-$

HER $2+$

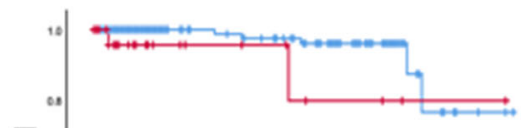

突

Overall $95 \% \mathrm{Cl}(147.6-167.3)$ $p$-value $=0.064$

OS
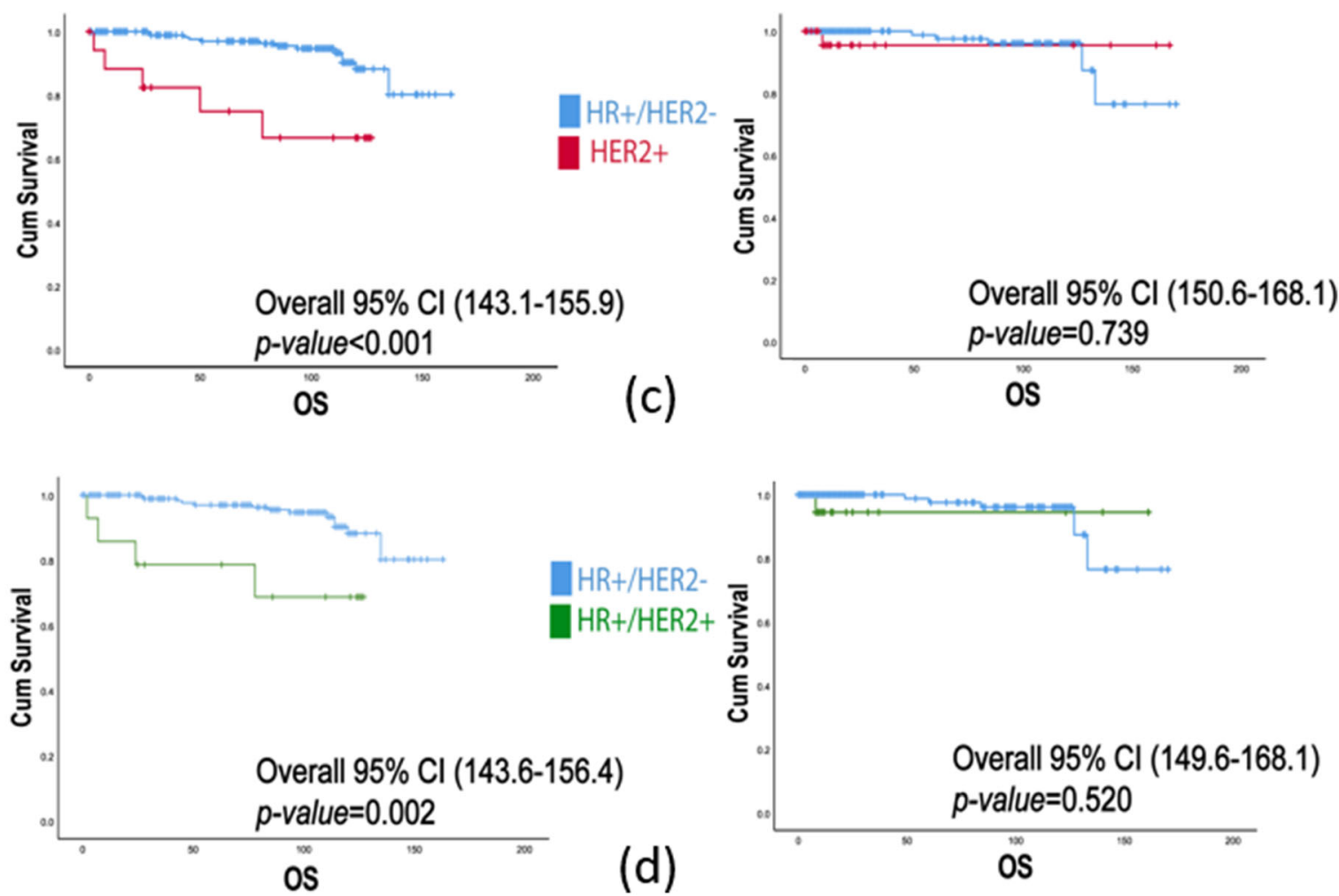

Fig. 4 Survival analysis according to the combined status of PTEN, HR, and HER2. The Y-axis shows the cumulative survival while the X-axis represents the months of overall survival. HR, hormone receptor; (OS) overall survival; (CI) confidence interval 
and HER2+ breast cancer according to the PTEN status can be related to the very biological functions of this tumor suppressor. Indeed, the PI3K/Akt/mTOR pathway is the most commonly upregulated pathway in HR- and HER2 + breast cancers, being involved in many aspects of cell growth, proliferation, survival, metabolism, and immune response regulation [40]. A deep disturbance of these processes is caused by abnormal activating events targeting PI3K/Akt, which leads to tumorigenesis, metastasis, tumor progression, and therapy resistance [36, 41-43]. In addition, PTEN activity in the nucleus is critical for tumor suppression due to the modulation of the DNA damage response and anti-tumor immune activity, independently of PTEN phosphatase activity [44-47]. These transversal biological roles might explain the implication of PTEN in the development of therapy resistance in breast cancer $[48,49]$. On the other hand, it has been proposed that loss of PTEN expression might be related to selective therapeutic pressure [50]. A recent study conducted on ER+ advanced breast cancer patients treated with a combination of the CDK4/6 inhibitor ribociclib and letrozole showed that loss of PTEN expression due to AKT activation could lead to the development of resistance to CDK4/6 inhibition [51]. Along with the observation that PTEN loss promotes resistance to PI3K $\alpha$ inhibitors, the authors highlight the possibility that one genetic event might prove sufficient for the same patient to develop clinical cross-resistance to multiple therapies, including anti-HER2 and ET [51].

This study has intrinsic limitations. First, given its retrospective nature and the long timeframe of patients' recruitment, it was not possible to unform the cohort for the treatment received. This could have led to an overestimation of PTEN independence as a prognostic biomarker, particularly in the trastuzumab-treated HER2 cohort. Indeed, a small percentage of patients with HER2+ breast cancer may not have received targeted adjuvant therapy, as before 2006 it was not approved in our Institution. Therefore, we cannot rule out the possibility that the lack of correlation of PTEN-WT and worse prognosis in HER2+ breast cancers could be related to a carry-over effect of the treatment with antiHER2 drugs, in particular considering the different protocols adopted during. However, this correlation was statistically significant in the PTEN-L group. To this end, functional studies exploring the specific role of antiHER2 drugs in PTEN-L breast cancer would be needed. Second, the relatively small number of tumors analyzed might have affected the ability to find additional correlations between PTEN and other significant clinicopathologic features. This study, however, should be considered hypothesis-generating. Further investigations in wider independent cohorts, with comprehensive molecular data and biostatistical analyses, are warranted to validate the clinical role of PTEN testing in breast cancer. Despite these limitations, this study offers novel insights on the potential clinical utility of combined PTEN, HER2, and HR testing for the identification of patients with high-risk breast cancer.

\section{Conclusions}

In conclusion, decreased expression of PTEN at the protein level is seen in almost half of breast cancer patients. We found a positive correlation between PTEN protein expression with HR and HER2 status and by the decreased relative expression of PTEN, both HR- and HER2 overexpression/amplification were significantly related to worse OS compared to the HR+/HER2- status. Moreover, this HER2 positivity either alone or concomitantly with HR positivity was associated with poorer survival compared to the HR+/HER2- status. Hence, the combined analysis of PTEN, HR, and HER2 may provide additional data to perform a tailored risk assessment while evaluating patients with breast cancers.

\begin{abstract}
Abbreviations
PTEN: Phosphatase and tensin homolog; PI3K: Phosphatidylinositol-3-kinase; HR: Hormone receptors; BCIRG: Breast Cancer International Research Group; WHO: World Health Organization; AJCC: American Joint Committee on Cancer; FFPE: Formalin-fixed, paraffin-embedded; TMA: Tissue microarray; ER: Estrogen receptor; PgR: Progesterone receptor; CAP: College of American Pathologists; PTEN-L: PTEN-low; PTEN-WT: PTEN-retained; TCGA: The Cancer Genome Atlas Network; OR: Odds ratio; Cl: Confidence interval; SD: Standard deviation; OS: Overall survival
\end{abstract}

\section{Supplementary Information}

The online version contains supplementary material available at https://doi. org/10.1186/s12885-021-08889-z.

Additional file 1: Figure S1. Overall survival according to the PTEN status. Figure S2. Survival analysis according to the combined status of PTEN, HR, and HER2. The Y-axis shows the cumulative survival while the $X$-axis represents the months of disease-free survival.

Additional file 2: Table S1. List of antibodies, clones, dilutions, antigen retrieval methods, and scoring systems adopted for

immunohistochemical analyses. Table S2. Bivariate analysis showing the association of combined assessment of PTEN-low and HR and HER2 markers with patients' death. Table S3. The demographic and clinical characteristics of all PTEN, HR and HER2 expression comparison.

\section{Acknowledgments}

The authors acknowledge support from the University of Milan through the APC initiative.

\section{Authors' contributions}

Conceptualization, N.F.; methodology, E.S., K.V., N.F.; software, E.S., K.V., and R.P.; validation, C.B., L.R., D.G., and E.G.R.; formal analysis, E.S., K.V., and N.F.; investigation, E.S., K.V., and N.F.; resources, S.F. and N.F.; data curation, E.S.; writing-original draft preparation, E.S. and K.V.; writing-review and editing, R.P., E.G.R. and N.F.; visualization, E.S., K.V., R.P., and N.F.; supervision, N.F.; project administration, N.F. All authors have read and agreed to the published version of the manuscript.

Funding

This research received no external funding. 


\section{Availability of data and materials}

The data presented in this study are available on request from the corresponding author. Publicly available datasets were analyzed in this study. This data can be found at https://www.cbioportal.org/.

\section{Declarations}

\section{Ethics approval and consent to participate}

The study was conducted according to the guidelines of the Declaration of Helsinki and approved by the Institutional Review Board of Fondazione IRCC S Ca' Granda - Ospedale Maggiore Policlinico (protocol code \#620_2018bis). Informed written consent was obtained from all subjects involved in the study.

\section{Consent for publication}

Not applicable.

\section{Competing interests}

E.G.R. has received advisory fees from Thermo Fisher Scientific and Novartis; honoraria from Thermo Fisher Scientific, Novartis, AstraZeneca, Roche; travel accommodation and expenses from Thermo Fisher Scientific, AstraZeneca, Roche, Novartis, Biocartis, Illumina. N.F. has received honoraria for consulting, advisory role, and/or speaker bureau from Merck Sharp \& Dohme (MSD), Boehringer Ingelheim, and Novartis. These companies had no role in the design of the study; in the collection, analyses, or interpretation of data; in the writing of the manuscript, and/or in the decision to publish the results. All other authors declare no potential conflicts of interest.

\section{Author details}

'Department of Oncology and Hemato-Oncology, University of Milan, Via Festa del Perdono 7, 20122 Milan, Italy. ${ }^{2}$ Division of Pathology, IEO, European Institute of Oncology IRCCS, Via Giuseppe Ripamonti 435, 20141 Milan, Italy. ${ }^{3}$ Division of Medical Oncology, Fondazione IRCCS Ca' Granda - Ospedale Maggiore Policlinico, Via Francesco Sforza 35, 20122 Milan, Italy. ${ }^{4}$ Breast Surgery Unit, Fondazione IRCCS Ca' Granda - Ospedale Maggiore Policlinico, Via Francesco Sforza 35, 20122 Milan, Italy. Division of Pathology, Fondazione IRCCS Ca' Granda - Ospedale Maggiore Policlinico, Via Francesco Sforza 35, 20122 Milan, Italy. ${ }^{6}$ Department of Biomedical, Surgical, and Dental Sciences, University of Milan, Via della Commenda 10, 20122 Milan, Italy.

\section{Received: 1 May 2021 Accepted: 19 October 2021}

\section{Published online: 28 October 2021}

\section{References}

1. Masson GR, Williams RL. Structural Mechanisms of PTEN Regulation. Cold Spring Harb Perspect Med. 2020;10(3). https://doi.org/10.1101/cshperspect.a 036152

2. Fusco N, Malapelle U, Fassan M, Marchiò C, Buglioni S, Zupo S, et al. PIK3CA Mutations as a Molecular Target for Hormone Receptor-Positive, HER2Negative Metastatic Breast Cancer. Front Oncol. 2021;11(562). https://doi. org/10.3389/fonc.2021.644737.

3. Carbognin L, Miglietta F, Paris I, Dieci MV. Prognostic and Predictive Implications of PTEN in Breast Cancer: Unfulfilled Promises but Intriguing Perspectives. Cancers (Basel). 2019;11(9). https://doi.org/10.3390/cancers11 091401

4. Lopez G, Noale M, Corti C, Gaudioso G, Sajjadi E, Venetis K, et al. PTEN Expression as a Complementary Biomarker for Mismatch Repair Testing in Breast Cancer. Int J Mol Sci. 2020;21(4). https://doi.org/10.3390/ijms210414 61.

5. Vidwans SJ, Turski ML, Janku F, Garrido-Laguna I, Munoz J, Schwab R, et al. A framework for genomic biomarker actionability and its use in clinical decision making. Oncoscience. 2014;1(10):614-23. https://doi.org/10.18632/ oncoscience.90.

6. Bazzichetto C, Conciatori F, Pallocca M, Falcone I, Fanciulli M, Cognetti F, et al. PTEN as a Prognostic/Predictive Biomarker in Cancer: An Unfulfilled Promise? Cancers (Basel). 2019;11(4):435

7. Kingston B, Bailleux C, Delaloge S, Schiavon G, Scott V, Lacroix-Triki M, et al. Exceptional response to AKT inhibition in patients with breast Cancer and germline PTEN mutations. JCO Precision Oncol. 2019;(3):1-7. https://doi. org/10.1200/PO.19.00130
8. Fabi A, Metro G, Di Benedetto A, Nisticò C, Vici P, Melucci E, et al. Clinical significance of PTEN and p-Akt co-expression in HER2-positive metastatic breast cancer patients treated with trastuzumab-based therapies. Oncology. 2010:78(2):141-9. https://doi.org/10.1159/000312656.

9. Sajjadi E, Venetis K, Piciotti R, Invernizzi M, Guerini-Rocco E, Haricharan S, et al. Mismatch repair-deficient hormone receptor-positive breast cancers: biology and pathological characterization. Cancer Cell Int. 2021;21(1):266. https://doi.org/10.1186/s12935-021-01976-y.

10. Stern HM, Gardner H, Burzykowski T, Elatre W, O'Brien C, Lackner MR, et al. PTEN loss is associated with worse outcome in HER2-amplified breast Cancer patients but is not associated with Trastuzumab resistance. Clin Cancer Res. 2015;21 (9):2065-74. https://doi.org/10.1158/1 078-0432.CCR-14-2993.

11. Li S, Shen Y, Wang M, Yang J, LV M, Li P, et al. Loss of PTEN expression in breast cancer: association with clinicopathological characteristics and prognosis. Oncotarget. 2017;8(19):32043-54. https://doi.org/10.18632/oncota rget.16761.

12. Perez EA, Dueck AC, McCullough AE, Chen B, Geiger XJ, Jenkins RB, et al. Impact of PTEN protein expression on benefit from adjuvant trastuzumab in early-stage human epidermal growth factor receptor 2-positive breast cancer in the north central Cancer treatment group N9831 trial. J Clin Oncol. 2013;31(17):2115-22. https://doi.org/10.1200/JCO.2012.42.2642.

13. Nuciforo PG, Aura C, Holmes E, Prudkin L, Jimenez J, Martinez P, et al. Benefit to neoadjuvant anti-human epidermal growth factor receptor 2 (HER2)-targeted therapies in HER2-positive primary breast cancer is independent of phosphatase and tensin homolog deleted from chromosome 10 (PTEN) status. Ann Oncol. 2015;26(7):1494-500. https://doi. org/10.1093/annonc/mdv175.

14. Fusco N, Sajjadi E, Venetis K, Gaudioso G, Lopez G, Corti C, et al. PTEN alterations and their role in Cancer management: are we making headway on precision medicine? Genes. 2020;11(7):719. https://doi.org/10.3390/ genes 11070719

15. Fusco N, Lopez G, Corti C, Pesenti C, Colapietro P, Ercoli G, et al. Mismatch Repair Protein Loss as a Prognostic and Predictive Biomarker in Breast Cancers Regardless of Microsatellite Instability. JNCI Cancer Spectr. 2018;2(4): pky056.

16. Tan PH, Ellis I, Allison K, Brogi E, Fox SB, Lakhani S, et al. The 2019 World Health Organization classification of tumours of the breast. Histopathology. 2020;77(2):181-5. https://doi.org/10.1111/his.14091.

17. Rakha EA, El-Sayed ME, Lee AH, Elston CW, Grainge MJ, Hodi Z, et al. Prognostic significance of Nottingham histologic grade in invasive breast carcinoma. J Clin Oncol. 2008;26(19):3153-8. https://doi.org/10.1200/JCO.2 007.15.5986.

18. Amin MB, Edge SB, Greene FL, Byrd DR, Brookland RK, Washington MK, et al. AJCC Cancer staging manual. 8th edn: Springer International Publishing; 2017. https://doi.org/10.1007/978-3-319-40618-3.

19. Ercoli G, Lopez G, Ciapponi C, Corti C, Despini L, Gambini D, et al. Building up a high-throughput screening platform to assess the heterogeneity of HER2 gene amplification in breast cancers. J Vis Exp. 2017;13(1):233-6. https://doi.org/10.3791/56686.

20. Sciarra A, Lopez G, Corti C, Runza L, Ercoli G, Bonometti A, et al. Columnar cell lesion and apocrine hyperplasia of the breast: is there a common origin? The Role of Prolactin-induced Protein. Appl Immunohistochem Mol Morphol. 2019;27(7):508-514. https://doi.org/10.1097/PAl.0000000000000604.

21. Nielsen TO, Leung SCY, Rimm DL, Dodson A, Acs B, Badve S, et al. Assessment of Ki67 in breast Cancer: updated recommendations from the international Ki67 in breast Cancer working group. JNCl: J Natl Cancer Institute. 2020;113(7):808-19. https://doi.org/10.1093/jnci/djaa201.

22. Sakr RA, Barbashina V, Morrogh M, Chandarlapaty S, Andrade VP, Arroyo CD, et al. Protocol for PTEN expression by immunohistochemistry in formalinfixed paraffin-embedded human breast carcinoma. Appl Immunohistochem Mol Morphol. 2010;18(4):371-4. https://doi.org/10.1097/PAl.0b013e3181 d50bd5.

23. Mingo J, Luna S, Gaafar A, Nunes-Xavier CE, Torices L, Mosteiro L, et al. Precise definition of PTEN C-terminal epitopes and its implications in clinical oncology. NPJ Precis Oncol. 2019;3(1):11. https://doi.org/10.1038/s41698-01 9-0083-4.

24. Cerami E, Gao J, Dogrusoz U, Gross BE, Sumer SO, Aksoy BA, et al. The cBio cancer genomics portal: an open platform for exploring multidimensional cancer genomics data. Cancer Discov. 2012;2(5):401-4. https://doi.org/10.11 58/2159-8290.CD-12-0095. 
25. Lopez G, Costanza J, Colleoni M, Fontana L, Ferrero S, Miozzo M, et al. Molecular Insights into the Classification of Luminal Breast Cancers: The Genomic Heterogeneity of Progesterone-Negative Tumors. Int J Mol Sci. 2019;20(3). https://doi.org/10.3390/ijms20030510.

26. Moher D, Hopewell S, Schulz KF, Montori V, Gøtzsche PC, Devereaux PJ, et al. CONSORT 2010 explanation and elaboration: updated guidelines for reporting parallel group randomised trials. J Clin Epidemiol. 2010;63(8):e137. https://doi.org/10.1016/j.jclinepi.2010.03.004.

27. Fusco N, Vaira V, Righi I, Sajjadi E, Venetis K, Lopez G, et al. Characterization of the immune microenvironment in malignant pleural mesothelioma reveals prognostic subgroups of patients. Lung Cancer. 2020;150:53-61. https://doi.org/10.1016/j.lungcan.2020.09.026.

28. Moher D, Hopewell S, Schulz KF, Montori V, Gøtzsche PC, Devereaux PJ, et al. CONSORT 2010 explanation and elaboration: updated guidelines for reporting parallel group randomised trials. Int J Surg. 2012;10(1):28-55. https://doi.org/10.1016/j.ijsu.2011.10.001.

29. Invernizzi M, Corti C, Lopez G, Michelotti A, Despini L, Gambini D, et al. Lymphovascular invasion and extranodal tumour extension are risk indicators of breast cancer related lymphoedema: an observational retrospective study with long-term follow-up. BMC Cancer. 2018;18(1):935. https://doi.org/10.1186/s12885-018-4851-2

30. Invernizzi M, Michelotti A, Noale M, Lopez G, Runza L, Giroda M, et al. Breast Cancer Systemic Treatments and Upper Limb Lymphedema: A RiskAssessment Platform Encompassing Tumor-Specific Pathological Features Reveals the Potential Role of Trastuzumab. J Clin Med. 2019;8(2):138.

31. Ngeow J, Eng C. PTEN in Hereditary and Sporadic Cancer. Cold Spring Harb Perspect Med. 2020;10(4). https://doi.org/10.1101/cshperspect.a036087.

32. Gozzelino L, De Santis MC, Gulluni F, Hirsch E, Martini M. PI(3,4)P2 signaling in Cancer and metabolism. Front Oncol. 2020;10:360. https://doi.org/10.33 89/fonc.2020.00360

33. Kechagioglou P, Papi RM, Provatopoulou X, Kalogera E, Papadimitriou E, Grigoropoulos P, et al. Tumor suppressor PTEN in breast cancer: heterozygosity, mutations and protein expression. Anticancer Res. 2014; 34(3):1387-400.

34. Jones N, Bonnet F, Sfar S, Lafitte M, Lafon D, Sierankowski G, et al. Comprehensive analysis of PTEN status in breast carcinomas. Int J Cancer. 2013;133(2):323-34. https://doi.org/10.1002/ijc.28021.

35. Beg S, Siraj AK, Prabhakaran S, Jehan Z, Ajarim D, Al-Dayel F, et al. Loss of PTEN expression is associated with aggressive behavior and poor prognosis in middle eastern triple-negative breast cancer. Breast Cancer Res Treat. 2015;151(3):541-53. https://doi.org/10.1007/s10549-015-3430-3.

36. Grizzi G, Ghidini M, Botticelli A, Tomasello G, Ghidini A, Grossi F, et al. Strategies for increasing the effectiveness of aromatase inhibitors in locally advanced breast Cancer: an evidence-based review on current options. Cancer Manag Res. 2020;12:675-86. https://doi.org/10.2147/CMAR.S202965.

37. Nicolini A, Ferrari P, Duffy MJ. Prognostic and predictive biomarkers in breast cancer: past, present and future. Semin Cancer Biol. 2018;52(Pt 1):5673. https://doi.org/10.1016/j.semcancer.2017.08.010.

38. Liang $Y$, Lin B, Ye Z, Chen S, Yu H, Chen C, et al. Triple-high expression of phosphatase and tensin homolog (PTEN), estrogen receptor (ER) and progesterone receptor (PR) may predict favorable prognosis for patients with type I endometrial carcinoma. J Cancer. 2020;11(6):1436-45. https://doi. org/10.7150/jca.33720.

39. Estrella JS, Broaddus RR, Mathews A, Milton DR, Yao JC, Wang H, et al. Progesterone receptor and PTEN expression predict survival in patients with low- and intermediate-grade pancreatic neuroendocrine tumors. Arch Pathol Lab Med. 2014;138(8):1027-36. https://doi.org/10.5858/arpa.2013-01 95-OA.

40. Hoxhaj G, Manning BD. The PI3K-AKT network at the interface of oncogenic signalling and cancer metabolism. Nat Rev Cancer. 2020;20(2):74-88. https:// doi.org/10.1038/s41568-019-0216-7.

41. Porta C, Paglino C, Mosca A. Targeting PI3K/Akt/mTOR signaling in Cancer. Front Oncol. 2014;4:64

42. Ramapriyan R, Caetano MS, Barsoumian HB, Mafra ACP, Zambalde EP, Menon $\mathrm{H}$, et al. Altered cancer metabolism in mechanisms of immunotherapy resistance. Pharmacol Ther. 2019;195:162-71. https://doi. org/10.1016/j.pharmthera.2018.11.004.

43. Venetis K, Piciotti R, Sajjadi E, Invernizzi M, Morganti S, Criscitiello C, et al. Breast Cancer with bone metastasis: molecular insights and clinical management. Cells. 2021;10(6):1377. https://doi.org/10.3390/cells10061377.
44. Milella M, Falcone I, Conciatori F, Cesta Incani U, Del Curatolo A, Inzerilli N, et al. PTEN: multiple functions in human malignant tumors. Front Oncol. 2015:5:24. https://doi.org/10.3389/fonc.2015.00024.

45. Naderali E, Khaki AA, Rad JS, Ali-Hemmati A, Rahmati M, Charoudeh HN Regulation and modulation of PTEN activity. Mol Biol Rep. 2018;45(6):286981. https://doi.org/10.1007/s11033-018-4321-6.

46. Song MS, Carracedo A, Salmena L, Song SJ, Egia A, Malumbres M, et al. Nuclear PTEN regulates the APC-CDH1 tumor-suppressive complex in a phosphatase-independent manner. Cell. 2011;144(2):187-99. https://doi. org/10.1016/j.cell.2010.12.020.

47. Pagni F, Guerini-Rocco E, Schultheis AM, Grazia G, Rijavec E, Ghidini M, et al Targeting Immune-Related Biological Processes in Solid Tumors: We do Need Biomarkers. Int J Mol Sci. 2019;20(21). https://doi.org/10.3390/ijms2021 5452

48. Razavi P, Dickler MN, Shah PD, Toy W, Brown DN, Won HH, et al. Alterations in PTEN and ESR1 promote clinical resistance to alpelisib plus aromatase inhibitors. Nat Cancer. 2020;1(4):382-93. https://doi.org/10.1038/s43018-0200047-1.

49. Fan X, Kraynak J, Knisely JPS, Formenti SC, Shen WH. PTEN as a Guardian of the genome: pathways and targets. Cold Spring Harb Perspect Med. 2020; 10(9). https://doi.org/10.1101/cshperspect.a036194.

50. Juric D, Castel P, Griffith M, Griffith $\mathrm{OL}$, Won HH, Ellis $\mathrm{H}$, et al. Convergent loss of PTEN leads to clinical resistance to a PI(3)Kalpha inhibitor. Nature. 2015;518(7538):240-4. https://doi.org/10.1038/nature13948.

51. Costa C, Wang Y, Ly A, Hosono Y, Murchie E, Walmsley CS, et al. PTEN loss mediates clinical cross-resistance to CDK4/6 and PI3Kalpha inhibitors in breast Cancer. Cancer Discov. 2020;10(1):72-85. https://doi.org/10.1158/21 59-8290.CD-18-0830.

\section{Publisher's Note}

Springer Nature remains neutral with regard to jurisdictional claims in published maps and institutional affiliations.

Ready to submit your research? Choose BMC and benefit from:

- fast, convenient online submission

- thorough peer review by experienced researchers in your field

- rapid publication on acceptance

- support for research data, including large and complex data types

- gold Open Access which fosters wider collaboration and increased citations

- maximum visibility for your research: over $100 \mathrm{M}$ website views per year

At BMC, research is always in progress.

Learn more biomedcentral.com/submissions 